\title{
Newer generation antidepressants for young people: real-life evidence needed
}

\author{
Haseena Hussain, Bernadka Dubicka \& Paul Wilkinson
}

COMMENTARY ON... COCHRANE CORNER ${ }^{\dagger}$

\begin{abstract}
SUMMARY
Major depressive disorder in children and adolescents is common and associated with significant morbidity and mortality. This 2012 meta-analysis by Hetrick et al shows statistically significant, but small, improvements in depressive symptom scores and probability of remission with second-generation antidepressants (SGAs) compared with placebo. SGAs lead to a small, but significant, increase in risk of suicidal thoughts/attempts compared with placebo. Patients included in the primary studies had milder depression, less psychiatric comorbidity and less suicidality than those normally seen in clinical practice in the UK's National Health Service. However, primary studies had significant methodological shortcomings. Therefore, caution is needed when trying to generalise results to clinical practice.
\end{abstract}

\section{DECLARATION OF INTEREST}

None

\section{Clinical setting}

Major depressive disorder in children and adolescents is common: the lifetime prevalence estimate in 13- to 18-year-olds is $11.0 \%$ and the 12 -month prevalence rate is $7.5 \%$. The prevalence tends to increase across adolescence (Avenevoli 2015). There are differences in the neurobiological correlates of depression in children/adolescents and in adults (Kaufman 2001). A notable finding is that severity of the index episode of major depressive disorder is greater with earlier age at onset (Zisook 2007). Poor outcomes in adulthood are associated with recurrent depressive episodes in adolescence. A recurrent course increases the risk for suicide and adverse psychological and social consequences (Wilson 2015). Psychiatric comorbidity is common, with more than half (52.1-88.5\%) of those with paediatric depression having another comorbid psychiatric disorder (March 2004; Goodyer 2008). A prospective casecontrol study showed that individuals who had had major depressive disorder on initial assessment in adolescence had a fivefold increased risk for a first suicide attempt compared with healthy individuals 10-15 years later (Weissmann 1999).

\section{Current pharmacological treatments}

First-generation antidepressants include tricyclic antidepressants and monoamine oxidase inhibitors (MAOIs). The second (newer) generation includes selective serotonin reuptake inhibitors (SSRIs), serotonin-noradrenaline reuptake inhibitors (SNRIs), noradrenaline reuptake inhibitors (NRIs), noradrenaline-dopamine reuptake inhibitors (NDRIs), noradrenaline-dopamine disinhibitors (NDDIs) and tetrayclic antidepressants (TeCAs). Newer generation antidepressants have fewer adverse effects than older (first-generation) drugs.

A meta-analysis of placebo-controlled trials of tricyclic antidepressants in children and adolescents conducted by Hazell et al (1995) showed a small, statistically significant difference between active treatment and placebo in adolescents only. However, tricyclics showed poor tolerability due to the high prevalence of adverse effects. The increased use of SSRIs in adolescents followed from the first controlled trials of their use for depression and obsessive-compulsive disorder (OCD) in the under-18s in the late 1990s (Vitiello 2006). However, in 2003, owing to concerns about safety, in particular suicide risk, the Committee on Safety of Medicines (CSM) in the UK indicated that the majority of SSRIs and venlafaxine were not suitable for patients under 18 years of age. In 2004, the US Food and Drug Administration (FDA) released a new warning relaying concerns that antidepressants may increase suicidal thinking and behaviour in depressed adolescents.

\section{Method}

This month's Cochrane Corner review (Hetrick 2012) is an update of an earlier review (Hetrick 2007) that extends the search to October 2011.
ROUND THE CORNER
Haseena Hussain is an academic clinical fellow in child and adolescent psychiatry at the University of Cambridge and East London NHS Foundation Trust. Bernadka Dubicka is an honorary reader in child psychiatry in the Faculty of Biology, Medicine and Health, University of Manchester, and a consultant psychiatrist with Lancashire Care NHS Foundation Trust, working at the Junction Adolescent Unit, Lancaster. Paul Wilkinson is a university lecturer and honorary consultant in child and adolescent psychiatry at the University of Cambridge and Cambridgeshire and Peterborough NHS Foundation Trust. Correspondence Dr Haseena Hussain, University of Cambridge, Developmental Psychiatry, Douglas House, 18b Trumpington Road, Cambridge CB2 8AH, UK. Email: hh448@medschl.cam.ac.uk

\section{Copyright and usage}

(C) The Royal College of Psychiatrists 2017

${ }^{\dagger}$ See p. 74, this issue. 
The population selected was children and adolescents 6 to 18 years of age who had received a primary diagnosis of depressive disorder from a clinician according to DSM (American Psychiatric Association 2000) or ICD (World Health Organization 2004) diagnostic criteria, from both in-patient and out-patient settings. Trials involving combined second-generation antidepressant medication and psychological therapy were not included in the review.

The updated review, which included newer classes of antidepressant, identified 530 trials; of these, 22 full-text articles were assessed for eligibility, yielding a total of 19 studies in qualitative synthesis. Outcomes measured were depression symptom severity, remission or response, functioning, suicide-related outcomes, overall adverse outcomes and completion of trial protocol. Data from the FDA report of suiciderelated outcomes (Hammad 2004) were used in the review and, where this was not possible, individual trial reports were used instead. The Cochrane Collaboration's new 'risk of bias' tool assessed domains of sequence generation, allocation concealment, masking ('blinding') of participants and assessors, incomplete outcome data and selective reporting. Treatment effects were measured using risk ratios (RR) for dichotomous outcomes and mean difference (m.d.) for continuous outcomes (using the Children's Depression Rating Scale - Revised (CDRS-R) for depressive symptoms). Most of the trials dealt with missing data using last observation carried forward (LOCF). Owing to expected heterogeneity between medications, a random-effects model was used for data synthesis.

\section{Results}

With the exception of 3 trials, all of the 19 reviewed were multicentre and most had two arms, comparing a newer generation antidepressant with placebo for a treatment period of between 6 and 12 weeks. The mean age ranged from 11.5 to 13.3 years in the child trials and 14.4 to 16.0 years in the adolescent trials. Trials of children and adolescents with comorbid psychiatric conditions were included only if data could be analysed separately. If there were concerns about organic brain injury or significant medical conditions potentially affecting the ability of patients to participate, these studies were also excluded. Trials including children and adolescents with an IQ of $\leq 70$ were excluded. Within the individual studies included, there was some variability in terms of exclusion criteria for psychiatric comorbidities, substance misuse and suicidality; 14 studies excluded participants on the basis of suicide risk. Exclusion of suicidality was not specifically stated in 3 studies and there was limited definition of suicide risk in 3 studies.

\section{Quality}

The risk of bias (Box 1) was unclear in many trials (allocation concealment, masking), and high in others because of incomplete outcome data and selective reporting. None of the trials included detailed reports on allocation concealment. All of the trials mentioned that they were 'double-blind', but there was little description of the masking in 10 of them. There were variable attrition rates between the control groups (11-82\%) and intervention groups (14-58\%), and the difference between treatment arms was most notable for the fluoxetine trials. Three trials (all paroxetine) listed an attempt to measure adherence to the intervention by pill count, but there was no mention of adherence in 11 trials. Receipt of additional therapy or support within trials was variable, with only some trials giving details of this.

\section{Efficacy of newer generation antidepressants}

Analysis of outcome data on depressive symptom severity revealed that those treated with a newer generation antidepressant had a small, statistically significant reduction in severity scores compared with placebo (14 trials; $N=2490$; CDRS-R m.d. $=-3.51$; $95 \%$ CI -4.55 to -2.47 ). Subgroup analyses of antidepressant $\mathrm{v}$. placebo by individual drug showed a statistically significant reduction in depression symptoms for those taking fluoxetine

BOX 1 Selection bias

In epidemiological studies, we are interested in estimating the association between an exposure (e.g. treatment) and outcome (e.g. depressive symptoms). Bias is systematic error in either the design or conduct of a study, resulting in incorrect estimates of the association between exposure and outcome. In selection bias, samples are not representative of the intended population.

In treatment studies, patients dropping out of studies early is called attrition bias. This is a type of selection bias because people who drop out of a study are likely to be different from those who remain in it - thus, the final sample is systematically non-representative of the baseline sample. This is a larger problem if drop-out rates differ between treatment arms.

Some trials used in this updated meta-analysis (Hetrick 2012) had large attrition rates that differed between intervention and control groups. This selection bias means that effect size estimates are likely to be incorrect. 
(3 trials; m.d. $=-5.63 ; 95 \%$ CI -7.39 to -3.86 ), sertraline (2 trials; m.d. $=-3.52 ; 95 \% \mathrm{CI}-6.64$ to -0.40 ) and escitalopram (2 trials; m.d. $=-2.67$; $95 \%$ CI -4.85 to -0.48 ). Unlike in the earlier metaanalysis (Hetrick 2007), there was no statistical heterogeneity of drug-placebo difference in depressive symptom improvement.

Data from the 16 trials (total of 2924 participants) reporting on remission showed a statistically significant increase in probability of remission when being treated with an antidepressant (RR 1.18; 95\% CI 1.08-1.28) compared with placebo. There was also evidence from 9 trials of antidepressant treatment improving function when compared with placebo $(N=1593 ;$ m.d. $=2.20$; 95\% CI 0.90-3.49).

\section{Adverse events}

Seventeen trials had data on suicide-related outcomes, showing suicide thoughts/attempts in $5.3 \%$ of those on newer generation antidepressants compared with $3.3 \%$ on placebo, a statistically significant difference. Adverse outcomes were reported differently in each trial, with 11 trials listing participants experiencing any adverse event and some trials not including data on adverse outcomes.

\section{Exploring forest plots}

The results of a meta-analysis can be displayed as a forest plot, and Fig. 1 shows an example from the review. Although the graph is reproduced at reduced size, it serves to show key features of such plots. There are 14 studies included for 7 newer generation antidepressants that have been compared with placebo. The forest plot has one horizontal line representing each study in the meta-analysis, plotted on the $y$-axis. The $x$-axis represents the difference between treatment groups. In this case, this represents the mean difference in CDRS-R scores (a measure of severity of depressive symptoms). Sometimes this is the standardised mean difference (difference in means divided by standard deviation, which is easier to interpret) or risk ratio for remission. The $x$-value for the black box on each line shows the mean difference for that study and is drawn in proportion to the weight that individual study has in the metaanalysis. The horizontal line represents the 95\% CI. A vertical line is plotted at the point of no effect on the $x$-axis (zero in this case). This makes it easy to tell which studies are statistically significant (as the confidence intervals would not include the point of no effect). Pooled differences for the studies are given as a diamond: in this case this is given for each group of studies, but it can also be given for each antidepressant. The fattest point of the diamond represents the mean difference, with the horizontal range representing the $95 \%$ CI. As these pooled effects include all studies, sample size is by nature larger, so confidence intervals are narrower.

The first line represents the Emslie 2006 study (published and unpublished data: see Cochrane review for details). The mean difference in CDRS-R is +0.80 , indicating that patients given placebo had more improvement than those given paroxetine. As the confidence interval includes 0 , this was not statistically significant. Of note, all three fluoxetine studies demonstrated fluoxetine to be better than placebo and had confidence intervals that did not include zero. The black diamond at the bottom of the forest plot shows the average mean difference of -3.51 (95\% CI -4.55 to -2.47$)$. This suggests that those given antidepressants have a slightly better outcome than those given placebo, and that this is statistically significant, therefore it is unlikely to be a chance finding.

\section{Discussion}

The overall results of this Cochrane review show that depressed adolescents improve more from taking newer generation antidepressants than placebo. The difference is small, but it is statistically significant. The meta-analysis was not designed to directly compare the different antidepressants. Looking at individual drug groups, there was a small effect size when treated with fluoxetine, sertraline or escitalopram when compared with placebo. Of these antidepressants, fluoxetine showed the greatest effect size when compared with placebo. There was no evidence that the difference in relative efficacy between antidepressants is more than would be expected by chance (as measured by statistical heterogeneity $\chi^{2}$ ).

Drug-placebo differences in these trials of antidepressants for paediatric depression were smaller than in the Cochrane meta-analysis of antidepressants for paediatric anxiety disorders/ OCD (Vitiello 2006), probably because the placebo response rate is higher for depression (Ipser 2009). The trials for depression excluded children/adolescents with severe depression or suicidality, mainly for safety reasons. For example, the seminal TADS trial (March 2004) excluded adolescents with active suicidality and those who had attempted suicide within the previous 6 months. However, placebo response decreases with increasing severity of the depressive episode (Bridge 2009), therefore antidepressant-placebo differences may be larger and more clinically 
Review: Newer generation antidepressants for depressive disorders in children and adolescents Comparison: 1 Newer generation antidepressant versus placebo (by drug)

Outcome: 1 Depressive symptom severity (CDRS-R)

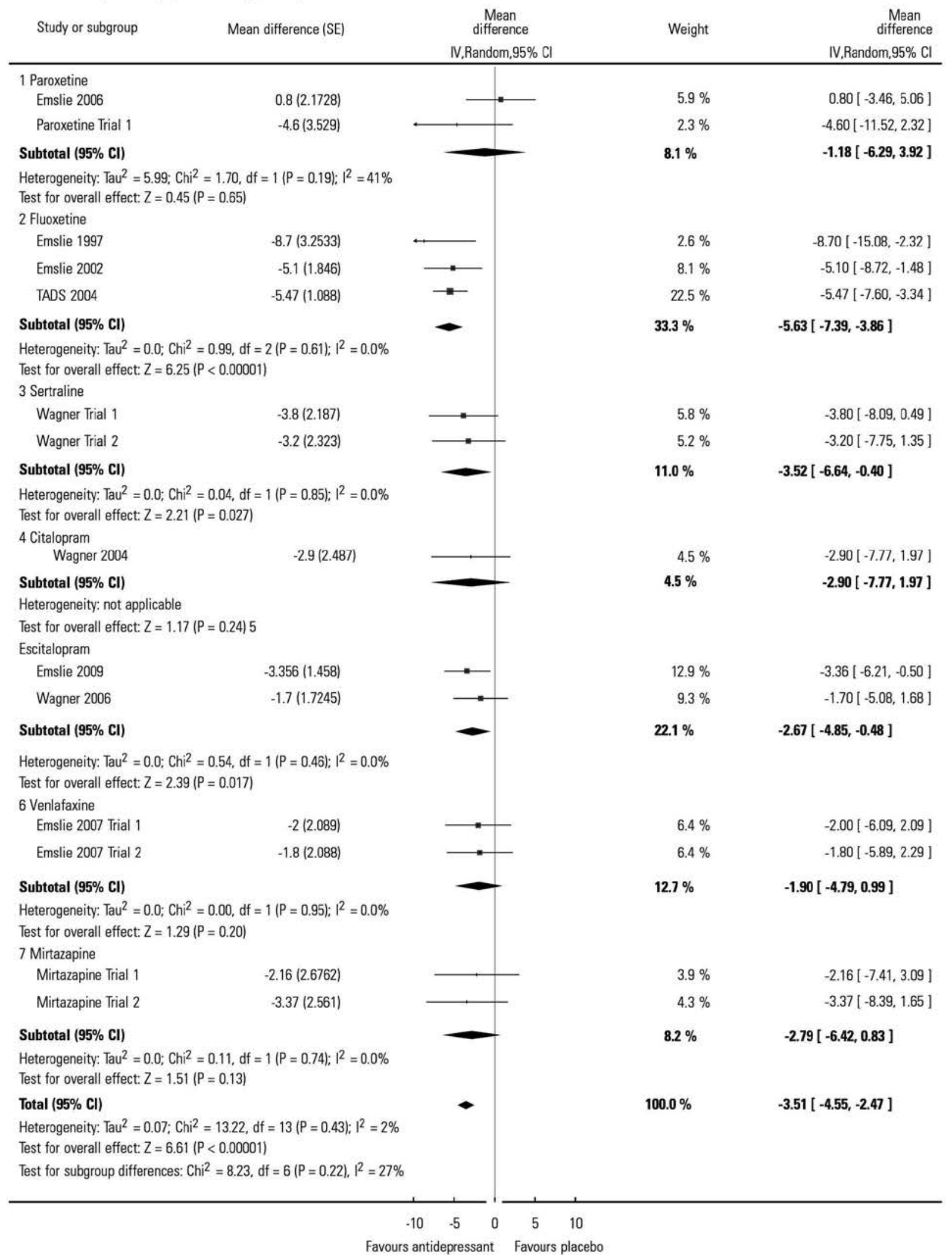

FIG 1 Comparison of newer generation antidepressants v. placebo (by drug) for the outcome depressive symptom severity, measured using the Children's Depression Rating Scale - Revised (CDRS-R); see the Cochrane review for study details. From Hetrick et al (2012): Analysis 1.1. Comparison 1; (C) 2012 The Cochrane Collaboration. Published by John Wiley \& Sons, Ltd. 
significant in young people with severe depression. High placebo response rates mean that the act of giving a placebo may be therapeutic - this should not be ignored and is likely to contribute to treatment response from antidepressants (Nutt 2008). Fourteen studies in Hetrick et als review excluded participants on the basis of suicidality, although three of these did not expand on their definition of suicidality.

This meta-analysis confirmed that suicidal ideation/attempts are significantly more common in children and adolescents prescribed antidepressants compared with placebo. However, such events were rare because pre-existing suicidality was an exclusion criterion for the studies. It is therefore difficult to extrapolate whether the effects of antidepressants on suicidality are greater or smaller in those with pre-existing suicidality, and how representative these findings may be in a typical clinic population.

\section{Previous reviews and guidelines}

The original review by Hetrick et al (2007) included 12 trials to determine the efficacy and safety of SSRIs compared with placebo. They used data from 10 of the trials and found evidence in favour of treatment with antidepressant medication compared with placebo (RR 1.80, 95\% CI 1.19-2.72). Their more recent meta-analysis discussed here included newly added studies (on escitalopram, fluoxetine and paroxetine) and also results from non-SSRI second-generation antidepressants (mirtazapine and venlafaxine). Cipriani and associates in their meta-analysis of the comparative efficacy of antidepressants in children and adolescents with major depressive disorder found that only fluoxetine was statistically more effective than placebo (Cipriani 2016). Such a network meta-analysis may underestimate efficacy if medications included were compared only with placebo and there is no statistical heterogeneity (as happened with sertraline and escitalopram: R. Gibbons, personal communication, 2016).

The National Institute for Health and Care Excellence (2005, updated 2015) recommends that if SSRIs are to be used, fluoxetine should be used first line, with sertraline and citalopram as second-line options, despite these guidelines being updated after this 2012 Cochrane meta-analysis.

\section{Implications}

The results from this meta-analysis indicate that second-generation antidepressants lead to lower depressive symptom scores and a greater probability of remission compared with placebo. However, this effect is small. It appears to be largest for fluoxetine. There is also a small but significantly increased risk of suicidal ideation/ attempts from second-generation antidepressants compared with placebo. The authors conclude that these findings need to be interpreted with caution, owing to 'methodological shortcomings' of the primary studies. The application of this Cochrane review is limited because the exclusion criteria used in the trials mean that the population under study is not representative of many of the patients presenting to clinical services. Those seen in National Health Service (NHS) specialist child and adolescent services often differ from these study populations as they frequently have more severe impairment, comorbid psychiatric disorders (anxiety, substance use disorder, conduct disorder) and suicidal thoughts and behaviours (Dubicka 2010). Such patients tend to be excluded from most clinical trials.

This meta-analysis is in agreement with other meta-analyses/guidelines that fluoxetine has the best evidence of efficacy and so should be tried first. It suggests that sertraline and escitalopram should be tried second-line, although other articles reach different conclusions. The clinician needs to balance the risks of treating and not treating the depressive episode, on the basis of severity of illness, individual clinical need and risk of completed suicide from the disorder itself.

\section{References}

American Psychiatric Association (2000) Diagnostic and Statistical Manual of Mental Disorders (4th edn) (DSM-IV). APA.

Avenevoli S, Swendsen J, He J-P, et al (2015) Major depression in the National Comorbidity Survey-Adolescent Supplement: prevalence, correlates, and treatment. Journal of the American Academy of Child \& Adolescent Psychiatry, 54: 37-44.

Bridge J, Birmaher B, lyengar S, et al (2009) Placebo response in randomised controlled trials of antidepressants for pediatric major depressive disorder. American Journal of Psychiatry, 166: 42-9.

Cipriani A, Zhou X, Giovane C, et al (2016) Comparative efficacy and tolerability of antidepressants for major depressive disorder in children and adolescents: a network meta-analysis. Lancet, 388: 881-90.

Dubicka B, Elvins R, Roberts C, et al (2010) Combined treatment with cognitive-behavioural therapy in adolescent depression: meta-analysis. British Journal of Psychiatry, 197: 433-40.

Goodyer IM, Dubicka B, Wilkinson P, et al (2008) A randomised controlled trial of cognitive behaviour therapy in adolescents with major depression treated by selective serotonin reuptake inhibitors: the ADAPT trial. Health Technology Assessment, 12 (14): i-x, 1-60.

Hammad TA (2004) Relationship between Psychotropic Drugs and Pediatric Suicidality. US Food and Drug Administration.

Hazell P, O'Connell D, Heathcote D, et al (1995) Efficacy of tricyclic drugs in treating child and adolescent depression: a meta-analysis. $B M J, 310$ : 897-901.

Hetrick SE, Merry SN, McKenzie JE, et al (2007) Selective serotonin reuptake inhibitors (SSRIs) for depressive disorders in children and adolescents. Cochrane Database of Systematic Reviews, 3: CD004851 (doi: 10.1002/14651858.CD004851.pub2).

Hetrick SE, McKenzie JE, Cox GR, et al (2012) Newer generation antidepressants for depressive disorders in children and adolescents. 
Cochrane Database of Systematic Reviews, 11: CD004851 (doi: 10.1002/14651858.CD004851.pub3).

Ipser JC, Stein DJ, Hawkridge S, et al (2009) Pharmacotherapy for anxiety disorders in children and adolescents (Review). Cochrane Database of Systematic Reviews, 3: CD005170 (doi: 10.1002/14651858.CD005170. pub2).

Kaufman J, Martin A, King R, et al (2001) Are child-, adolescent-, and adult-onset depression one and the same disorder? Biological Psychiatry, 49: 980-1001.

March J, Silva S, Petrycki S, et al (2004) Fluoxetine, cognitive-behavioral therapy, and their combination for adolescents with depression: Treatment for Adolescents with Depression Study (TADS) randomized controlled trial. JAMA, 292: 807-20.

National Institute for Health and Care Excellence (2005) Depression in Children and Young People: Identification and Management (Updated March 2015)(Clinical Guideline CG28). NICE.
Nutt DJ, Malizia AL (2008) Why does the world have such a 'down' on antidepressants? Journal of Psychopharmacology, 22: 223-6.

Vitiello B, Zuvekas S, Norquist G (2006) National estimates of antidepressant medication use among U.S. children, 1997-2002. Journal of the American Academy of Child \& Adolescent Psychiatry, 45: 271-9.

Weissmann MM, Wolk S, Goldstein RB, et al (1999) Depressed adolescents grown up. JAMA, 281: 1707-13.

World Health Organization (2004) ICD-10: International Statistical Classification of Diseases and Related Health Problems (2nd edn) (vol. 3). WHO.

Wilson S, Hicks B, Foster K, et al (2015) Age of onset and course of major depressive disorder: associations with psychosocial functioning outcomes in adulthood. Psychological Medicine, 45: 505-14.

Zisook S, Lesser I, Stewart JW, et al (2007) Effect of age at onset on the course of major depressive disorder. American Journal of Psychiatry, 164: 1539-46. 